\title{
Quality in pancreatic endoscopic ultrasound: what's new in 2020?
}

\author{
loannis S. Papanikolaou ${ }^{a}$, Georgios Tziatzios ${ }^{\mathrm{a}}$, Pantelis S. Karatzas ${ }^{\mathrm{b}^{*}}$, Paraskevas Gkolfakis ${ }^{\mathrm{c}^{*}}$, \\ Antonio Facciorusso ${ }^{\text {, }}$, Konstantinos Triantafyllou ${ }^{\mathrm{a}}$
}

National and Kapodistrian University of Athens, "Attikon" University General Hospital, Athens, Greece; Laiko General Hospital, University of Athens, Medical School, Athens, Greece; Erasme University Hospital, Université Libre de Bruxelles, Brussels, Belgium; University of Foggia AOU, Foggia, Italy

\begin{abstract}
Quality assessment and improvement of an endoscopic service has emerged as a basic component of everyday gastrointestinal endoscopy. In order to ensure a high level of quality, a series of actions must be adopted when performing an endoscopic examination. Nonetheless, quality still remains a qualitative parameter; thus, implementation of specific indicators of quality is warranted. Irrespective of the nature of the endoscopic procedure, quality indicators usually refer to either structural properties of an endoscopy unit (e.g., examination availability), procedural factors (e.g., diagnostic accuracy), or patient outcomes (e.g., occurrence of an adverse event related to performance of an endoscopic procedure). Moreover, they are usually classified into 3 distinct sections, according to the phase of the procedure they relate to: i.e., before, during, and after the examination. The aim of this review is to present measures that need to be adopted in order to reach an optimal quality level during an endoscopic ultrasound examination and to provide upto-date data regarding the respective quality indicators implicated.
\end{abstract}

Keywords Endoscopic, ultrasound, quality, quality indicators

Ann Gastroenterol 2020; 33 (6): 1-7

\section{Introduction}

Endoscopic ultrasound (EUS) remains the cornerstone of the diagnostic and staging algorithm for various lesions of the gastrointestinal (GI) tract, the abdomen and the mediastinum,

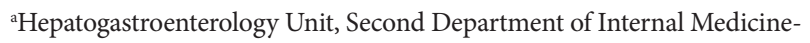
Propaedeutic, Research Institute and Diabetes Center, National and Kapodistrian University of Athens, "Attikon" University General Hospital, Athens, Greece (Ioannis S. Papanikolaou, Georgios Tziatzios,

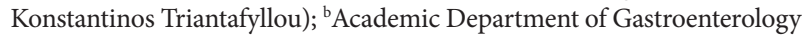
and Hepatology, Laiko General Hospital, University of Athens, Medical School, Athens, Greece (Pantelis S. Karatzas); 'Department of Gastroenterology Hepatopancreatology and Digestive Oncology, Erasme University Hospital, Université Libre de Bruxelles, Brussels, Belgium (Paraskevas Gkolfakis); 'Gastroenterology Unit, Department of Medical Sciences, University of Foggia AOU, Foggia, Italy (Antonio Facciorusso) ${ }^{*}$ They are equally contributed to this work

Conflict of Interest: None

Correspondence to: Ioannis S. Papanikolaou, MD, $\mathrm{PhD}$, Associate Professor of Gastroenterology and Internal Medicine, Hepatogastroenterology Unit, Second Department of Internal Medicine - Propaedeutic, Research Institute and Diabetes Center, Medical School, National and Kapodistrian University of Athens, "Attikon" University General Hospital, 1, Rimini Street, 12462 Athens, Greece, e-mail: ispapn@hotmail.com

Received 26 June 2020; accepted 18 August 2020; published online 2 October 2020

DOI: https://doi.org/10.20524/aog.2020.0537 assisting in the preoperative staging and restaging of GI tumors. The introduction of endoscopic ultrasound-guided fine-needle aspiration (EUS-FNA), revolutionized the examination's nature, adding an interventional aspect to its diagnostic core, allowing acquisition of tissue samples even from lesions outside the GI tract (e.g., in the pancreas). This particular feature differentiates EUS from other diagnostic modalities, including computed tomography or magnetic resonance imaging (MRI) [1]. On the other hand, quality indicators have been integrated into everyday GI endoscopy (GIE) practice, aiming to improve endoscopic services, each time taking into account the specific aspects of each patient's disease and pursuing the ultimate goal: to provide optimal medical care [2]. In order to clarify the essence of quality, the Department of Health and Human Services Institute of Medicine (USA), has defined it as "the degree to which healthcare services for individuals and populations increase the likelihood of desired health outcomes and are consistent with current professional knowledge" [3]. During recent years, quality has undergone significant changes, evolving over time into a significant prerequisite that should be met in all endoscopic procedures, implicating not only patients and physicians but also the services of healthcare systems. Interestingly, even among these 3 pillars, quality has a completely different orientation: patients desire to receive high-quality services regardless of cost, whereas clinicians pursue the best possible management, combined with a minimum risk of associated complications, and healthcare systems adopt an internal policy to ensure that the quality of 
the services provided remains high. The American Society of Gastrointestinal Endoscopy (ASGE) published the first series of guidelines regarding quality measures in GIE, updated in 2015 [4]. Outside the USA, in a recent publication the European Society of Gastrointestinal Endoscopy (ESGE) has also highlighted quality in GIE, and particularly EUS [5].

Moreover, quality, being per se a qualitative parameter, renders any attempt at its objective evaluation rather problematic. Thus, there is a need for surrogate markers that will allow an accurate assessment of any measures taken to enhance GIE and EUS performance in particular. In order to achieve this goal, we have used the so-called quality indicators (QIs), which are tools that enable us to quantify our effort to ascertain quality by evaluating the efficacy of the quality measures implemented during the procedure or, as stated elsewhere, to allow performance of a comparison "between an individual or a group and an ideal or benchmark" [6,7]. QIs may reflect structural conditions of an endoscopy unit (e.g., examination availability), procedure-related factors (e.g., diagnostic accuracy of a given procedure), or final outcomes (e.g., an adverse event related to the procedure) [8]. They are usually classified according to the phase of the procedure with which they are related: pre-procedure, during the procedure and post-procedure (Fig. 1). This classification helps us comprehend the potential impact of the implementation of quality measures in the various aspects of the EUS examination. Approaching these measures, and their respective QIs, using this prism (i.e., pre-, during and post-endoscopy) also allows their critical appraisal, another issue addressed within this review.

\section{QIs in the pre-procedure phase}

This heading refers to all potential physician-patient interactions occurring prior to the beginning of the endoscopic procedure. In this phase, the following QIs are included:

\section{Indications for EUS}

As a first step, the physician must thoroughly inform patients regarding the procedure's indications, as well as the availability of alternative diagnostic modalities. Not only must an appropriate indication be included in a published standard list and be present in at least $80 \%$ of all EUS procedures performed in a endoscopic unit or by an individual, but this indication should also be clearly documented [8]. It should be noted that performing EUS for an indication outside those listed in the aforementioned literature may, under certain circumstances, be an acceptable strategy. However, in that case scenario, the patient should be offered a detailed explanation regarding the rationale that led to this decision and this should also be documented in the report $[9,10]$. In light of these statements, one apparent condition necessitating the performance (or avoidance) of EUS without strict adherence to an appropriate indication is the issue of local availability, which can exert significant impact on physicians' decisionmaking according to the availability of resources. For example, EUS could replace MRI when local availability dictates it, e.g., when assessment for potential vascular invasion of a pancreatic head mass comes into question. In case the lesion is deemed operable, EUS-FNA should not be performed, as advocated by the current literature, and the EUS should serve only for diagnosis and/or staging [1,2].

\section{Informed consent form}

This phase usually involves a detailed step-by-step discussion between patients and physicians regarding the EUS procedure (this should ideally be conducted for all endoscopic procedures, irrespective of their diagnostic or interventional nature, including informing patients about potential complications). All procedure stages, including

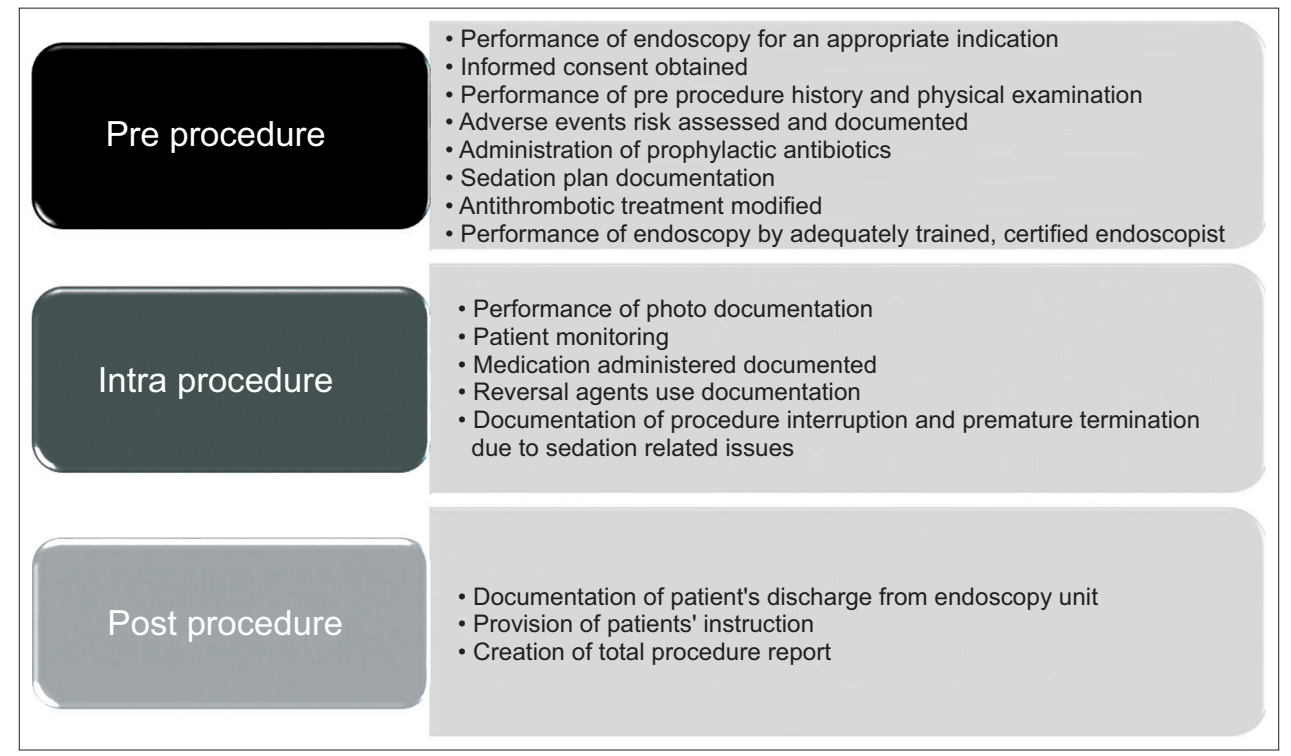

Figure 1 Classification of quality indicators regarding endoscopic ultrasound 
the merits and-equally significant-the potential caveats and complications of EUS, and particularly EUS-FNA, such as bleeding $(0-0.5 \%)$, infection $(<1 \%)$ and pancreatitis (0-2\%) should be discussed in detail, taking into account the patient's capability to truly comprehend the information provided [11,12]. Although the rates of tumor seeding [13-19] and perforation [20-22] are very low, the possibility of their rare occurrence does exist, and thus should be also known. The patient must not only be aware of all the information related to the examination, but, equally important, should be provided with enough time to raise queries before finally signing the informed consent form (ICF). It is important to note that withdrawal of consent should always be possible, and physicians should anticipate this by providing their patients enough time to do so if this is their wish. A signed ICF should be obtained in at least in $98 \%$ of cases [8]. In case specific EUS-techniques are planned, e.g., celiac plexus neurolysis or radiofrequency tumour ablation, additional explanations regarding the specific complications directly linked to these interventions should also be provided [2]. The endoscopist's level of expertise regarding the procedure is also another significant QI. Nowadays, patients deserve the right to know the level of expertise of the performing endoscopist, including his/her complication rate. Although the rate of examinations performed by a fully-trained and certified endoscopist to perform this procedure should reach the cutoff of $98 \%$ [8], an optimal and validated threshold that will define the precise level of expertise for EUS remains yet to be determined.

\section{Management of medications}

Physicians should always obtain an in-depth medical history, focusing particularly on the use of anticoagulant and/or antiplatelet medication. Specific questions regarding the exact type and dosage of these drugs need to be asked, in order to carry out the appropriate changes on time, prior to the procedure. In case of anticoagulant use, a vulnerable equilibrium must be preserved, where the patient is stratified according to the presumable risk of bleeding associated with the endoscopic procedure, weighed against the potential cardiovascular risk. Moreover, each endoscopic procedure is awarded a low or high risk for complications [12,23,24]. While EUS per se is considered a low-risk procedure, EUS with the addition of FNA is listed among the high-risk ones. When performing simple diagnostic EUS, anticoagulants can be safely continued, while only the morning dose on the day of the procedure should be skipped in the case of direct oral anticoagulants (DOACs). Patients receiving warfarin should have their international normalized ratio (INR) checked prior to the procedure; when this is within a therapeutic range the EUS procedure can be performed safely. On the contrary, in cases when EUS-FNA is planned, the severity of the underlying cardiovascular disease will guide the dosing modifications of anticoagulation and antiplatelets. Clopidogrel and prasugrel must be withdrawn 5 days prior to the examination provided the cardiovascular risk is deemed low. If this risk is considered high, the endoscopist should liaise with the responsible cardiologist to reach the best decision about the patient's favorable outcome. On the other hand, DOACs must be discontinued for $48 \mathrm{~h}$ (or $72 \mathrm{~h}$ in elderly patients with creatinine clearance $<30-50 \mathrm{~mL} /$ $\min$ ). Warfarin can be withheld until the INR returns to the normal range in case of low cardiovascular risk, or replaced by low molecular weight heparin, whenever the cardiovascular risk is high [25]. Puncture of cystic lesions is usually followed by antibiotic administration, acting as prophylaxis for infectious complications. Although this policy has been integrated into everyday worldwide clinical practice, its efficacy remains questionable, given the fact that the actual risk for infection remains very low (less than 1\%) [26]. The latest ASGE guidelines suggest the use of prophylactic antibiotics only in cases of EUSFNA of mediastinal and pancreatic cystic lesions; however, this was merely a weak recommendation, underlining the fact that data from prospective, randomized studies were lacking [27]. Indeed, the very first randomized trial evaluating the effect of antibiotic prophylaxis on the incidence of pancreatic cyst infection after EUS-FNA was only published very recently [26]. In this multicenter, randomized, non-inferiority trial conducted in Spain, prophylaxis with ciprofloxacin was compared against placebo administration in patients undergoing EUSFNA for pancreatic cystic lesions. Among the 226 patients randomized, only 1 patient (in the placebo group) developed an infection $(0.87 \%)$, while the incidence of infections did not differ significantly with or without ciprofloxacin prophylaxis. This finding is in line with the results reported in previous retrospective studies, all of which confirmed that antibiotic prophylaxis is not necessary [28-31]. In light of this emerging evidence, forthcoming guidelines should perhaps reappraise this particular statement. On the other hand, studies of the need for antibiotic prophylaxis before through-the-needle biopsy of pancreatic cystic lesions are warranted and welcome.

\section{Qls during the procedure}

This period is usually defined as extending from the time sedation is administrated until the endoscope is removed from the patient [2]. EUS is usually performed in the context of special indications, aiming to provide specific answers. Besides the delineation of subepithelial tumors, which is a major indication for EUS, though not of the pancreas, EUS is most frequently performed for tissue sampling and tumor staging, when pancreatobiliary malignancies are in question [32]. Therefore, QIs concerning this time interval involve successful lesion sampling rates, accurate malignancy staging and identification of all anatomical structures [8]. Adequate tissue sampling can be evaluated only by rates of successful lesion sampling. Diagnostic rates for malignant tumors should be at least $71 \%$ in the case of adenocarcinoma of the pancreas $[33,34]$ and even higher for nodal involvement (percentages up to $87 \%$ have been reported for nodal sampling in esophageal cancer) [35-37]. Although difficult to measure, the endoscopist's personal performance should be at least non-inferior to the abovementioned targets. To 
facilitate this, the ROSE (rapid-on site evaluation) method can be adopted, which involves an immediate in-room first evaluation of the samples by a present cytopathologist to ensure adequate material has been obtained. In case ROSE is not available, a minimum number of punctures from the lesion evaluated should be performed (e.g., 5-7 needle passes for pancreatic adenocarcinoma) [9]. However, the latter might be refuted by recent, real-world data suggesting that even 2 needle passes in EUS-FNA of solid pancreatic lesions can provide sufficient tissue to facilitate a diagnosis, with no significant incremental tissue yield if 3 passes are performed [38]. To make things even more challenging, evidence regarding the superiority of any specific technique for sampling pancreatic masses is currently limited and somewhat conflicting. In a recent network meta-analysis, no specific EUS-guided tissue sampling technique was found to be superior in terms of diagnostic accuracy, sample adequacy, or histologic procurement rate for solid pancreatic masses, irrespective of different needle types (FNA vs. fineneedle biopsy $[\mathrm{FNB}]$ ), or needle sizes (19-G vs. 22-G vs. 25-G) that were compared [39], although FNB has been proven to outperform standard FNA when it comes to the sampling of sub-epithelial lesions [40]. Thus, this statement is likely to be reappraised with the advent of newer FNB end-cutting needle designs (i.e., Acquire, Boston Scientific Corp, Natick, Massachusetts, United States or SharkCore, Medtronic, Minneapolis, Minnesota, United States) [41,42].

Another significant issue is tumour staging, where depth of invasion and presence of pathological lymph nodes must be adequately clarified. The TNM staging system should be always used and documented, as EUS is the optimal diagnostic modality to evaluate the " $\mathrm{T}$ " and " $\mathrm{N}$ " parameters of this classification, at the cost of low sensitivity for distant metastases (i.e., the "M" parameter) [43-45]. Documentation percentage of the relevant structures (those representing the target of each specific procedure) is the first QI. The endoscopist should be able to accurately recognize relevant structures in at least $98 \%$ of the cases and, if possible, to provide images that illustrate the findings (Fig. 2, 3A,B). Moreover, the use of the Doppler feature, with which modern electronic echoendoscopes are endowed, provides us with the possibility to better demonstrate such structures. For example, Doppler ultrasound can clearly differentiate a vessel from a dilated duct and thus provide reliable and non-questionable image documentation (Fig. 4A,B). As far as subepithelial masses are concerned (as stated above, this is a less common indication when it comes to the pancreas) the specific layer from which the lesion arises should always be identified and recorded in appropriate images. In these images, the size and specific morphological features of the mass under evaluation should be clearly delineated [8]. Provision for adequate sedation holds a cardinal role in any type of GIE, as it increases patient compliance, facilitating the performance of a detailed procedure, and contributes to better patient satisfaction and willingness to undergo endoscopic procedures. QIs regarding sedation include evaluation of the following parameters: frequency of routine vital signs monitoring during sedation,

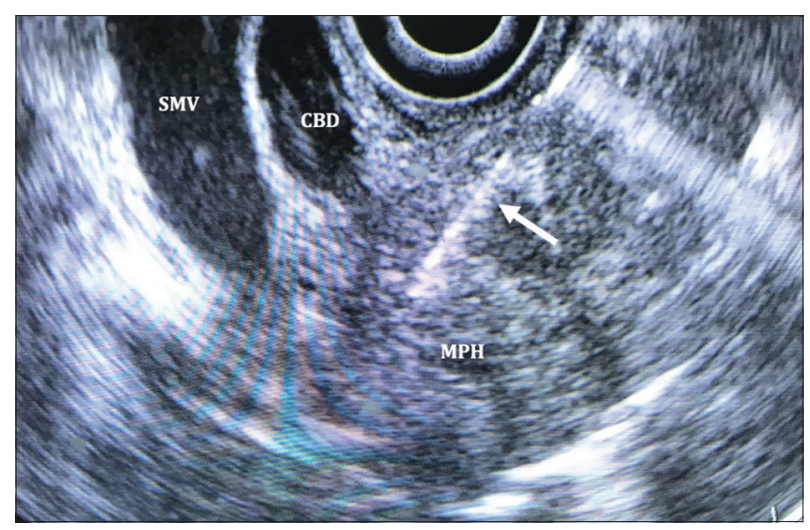

Figure 2 Endoscopic ultrasound-guided fine-needle aspiration of a mass in the head of the pancreas of a patient with lung cancer. The image clearly demonstrates the needle located within the mass (arrow), as well as relevant structures, including the mass in the head of the pancreas (MPH), the dilated common bile duct (CBD) and the superior mesenteric vein (SMV). Cytological examination of the acquired specimen revealed metastatic lung cancer
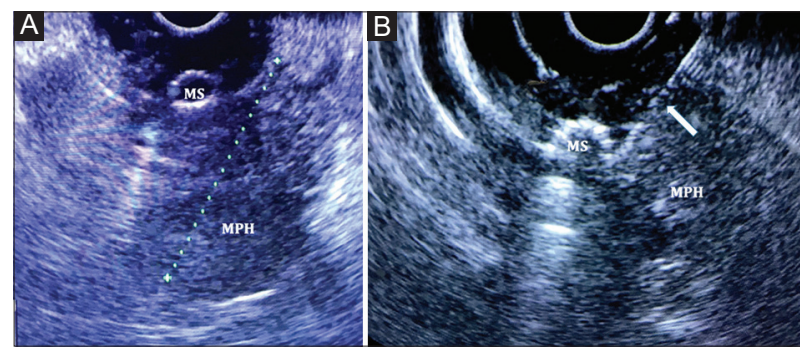

Figure 3 (A) Endoscopic ultrasound demonstrating a 3.5-cm hypogenic mass in the head of the pancreas (MPH). Because of obstructive jaundice, a metal stent (MS) had been placed in the patient's common bile duct a few days before. (B) Endoscopic ultrasound-guided fineneedle aspiration of the mass in Fig. 3A. Note the needle located within the mass (arrow), as well as relevant structures, including the mass (MPH) and the biliary metal stent (MS). Cytology of the acquired specimen revealed pancreatic adenocarcinoma
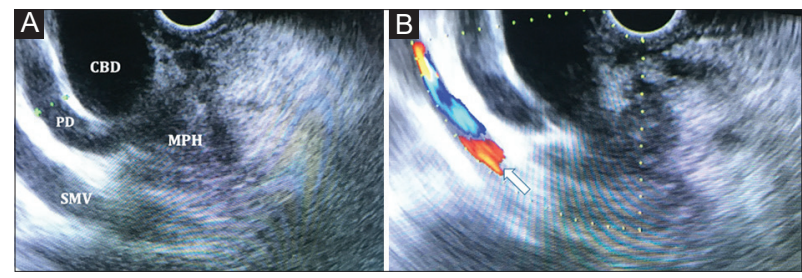

Figure 4 (A) Endoscopic ultrasound demonstrating a 2-cm hypogenic mass in the head of the pancreas (MPH) causing dilation of the common bile duct (CBD) and the pancreatic duct (PD) (doubleduct sign). Note the superior mesenteric vein (SMV), which is clearly differentiated from the dilated PD by the flow depicted in color (arrow) when Doppler ultrasound is used (B)

recording of dose and administration route of medication(s) being administered, use of reversal agents, and interruption or premature termination of the examination due to sedationderived complications [8]. 


\section{QIs after the procedure}

Several measures equally important to all previous QIs are included here, contributing to the procedure's quality: (a) identification and proper management of potential adverse events; (b) explaining the examination's findings to the patient and providing informative and adequate instructions for the direct post-endoscopy timeframe; (c) management of medications, and particularly antiplatelets/anticoagulants, after the examination; and (d) in case of histology/cytology acquisition, information regarding its follow up.

Adverse events related to EUS remain relatively rare, especially if no EUS-FNA or EUS-guided biopsy has been undertaken [12,46]. Nevertheless, endoscopists should always be able to recognize them promptly and deal with them effectively. The saying that "the worst complication in GIE is non-recognition or denial of a complication" seems to fit perfectly in this setting, and especially for pancreatobiliary GIE, including EUS: complications like bleeding and pancreatitis are usually mild and self-limiting, requiring no specific management. Although perforation seldom occurs, actions must be taken immediately, in order to ensure the best outcome for the patient $[47,48]$. As with all other endoscopies, patients must be informed in detail by the endoscopist regarding the examination's findings, as well as the impact these findings could have on their subsequent diagnostic and/ or therapeutic path. This measure may seem relatively simple and straightforward; however, existing evidence points in the opposite direction. Various reasons could be held accountable for this phenomenon, primarily linked to organizational factors; it is commonplace among large referral centers that, because of an immense workload, there is literarily " $n o$ time to explain" the findings to the patient. Moreover, the organization of healthcare systems could also contribute. For example, patients in several systems may be referred for GIE from general practitioners, who also theoretically carry the burden of informing the patient. This, however, can be rather burdensome for the non-specialist, especially when it involves providing further clarifications of complex and sophisticated examinations, as is the case for EUS. From the patient's point of view, rapid doctor-patient communication concerning the results of cytopathology or histology has demonstrated its value as an extremely important QI, even more significant than a good long-term relationship with the endoscopist [49]. However, real-world experience has shown that in everyday practice, things can be totally different. Moreover, providing the patient with fundamental postprocedural information is mandatory, including the avoidance of driving and/or intense physical activity, given their potentially fatal consequences. The vast majority of medications can usually be resumed safely in most cases of EUS or EUS-FNA after the examination, although when bleeding is suspected, following an individualized approach seems to be a sound practice $[50,51]$. As well as informing the patient about the procedure's findings, as mentioned above, the endoscopist also has the responsibility for receiving and interpreting the pathology/cytology results. As already outlined, EUS is performed with a specific clinical question in mind. The answer to this question can be rather challenging in many cases; for instance, EUS-FNA has a low negative predictive value for differentiating pancreatic cancer from chronic pancreatitis with a pseudotumoral mass, which reaches almost $73.9 \%$ [52]. Here, the endoscopist is the one who should be called upon to interpret the findings in a particular context and to decide whether repeating the negative EUS-FNA, recommending another diagnostic modality, or simply performing clinical follow up accompanied with imaging could be the best strategy in a given case. When a repeat procedure is decided upon, EUS-guided core biopsy, instead of "classical" EUS-FNA aiming at cytology sampling, could also be considered as an attractive alternative (e.g., in the aforementioned study, EUS-guided core biopsy led to an increase of the examination's negative predictive value up to $87 \%)$. Cooperation with the cytopathologist/pathologist, as well as other medical specialists involved in each and every case (radiologists, internists or surgeons) may also lead to improvement in the clinical decision. Finally, a QI that usually remains underrated is that of the patient's satisfaction. This QI is indeed a pivotal one, that in many cases is totally neglected by endoscopists or other physicians involved in the patient's management. This attitude however, should change and actually is being reconsidered. In fact, this QI not only refers to sedation and post-procedural pain issues, but at some point also reflects the effect of most of the quality measures applied during the entire procedure, as well as the general management by the attending physician $[53,54]$.

\section{Concluding remarks}

This review has presented a point-by-point description regarding the definition of the various quality measures that should be taken when performing EUS and has discussed the QIs related to them. Moreover, it has highlighted some of the most important ones, especially those most commonly used in everyday clinical practice. Clinicians should at all times keep in mind that these QIs are not part of a theoretical check-list for research studies only, but should rather be considered as a useful roadmap to guide us through uncharted areas of our everyday clinical practice, in an attempt to improve our patients' outcomes.

\section{References}

1. Papanikolaou IS, Fockens P, Hawes R, Rosch T. Update on endoscopic ultrasound: how much for imaging, needling, or therapy? Scand J Gastroenterol 2008;43:1416-1424.

2. Facciorusso A, Buccino RV, Muscatiello N. How to measure quality in endoscopic ultrasound. Ann Transl Med 2018;6:266.

3. Triantafyllou K, Michopoulos S, Hassan C. Quality in gastrointestinal endoscopy: the preface. Ann Transl Med 2018;6:258.

4. Rizk MK, Sawhney MS, Cohen J, et al. Quality indicators common to all GI endoscopic procedures. Gastrointest Endosc 2015;81:3-16.

5. Domagk D, Oppong KW, Aabakken L, et al. Performance measures 
for ERCP and endoscopic ultrasound: a European Society of Gastrointestinal Endoscopy (ESGE) Quality Improvement Initiative. Endoscopy 2018;50:1116-1127.

6. Hughes RG e. Advances in patient safety. In: Hughes RG, ed. Patient safety and quality: an evidence-based handbook for nurses. Rockville (MD): Agency for Healthcare Research and Quality (US), 2008.

7. Adler DG, Lieb JG 2nd, Cohen J, et al. Quality indicators for ERCP. Gastrointest Endosc 2015;81:54-66.

8. Wani S, Wallace MB, Cohen J, et al. Quality indicators for EUS. Am J Gastroenterol 2015;110:102-113.

9. Polkowski M, Jenssen C, Kaye P, et al. Technical aspects of endoscopic ultrasound (EUS)-guided sampling in gastroenterology: European Society of Gastrointestinal Endoscopy (ESGE) Technical Guideline - March 2017. Endoscopy 2017;49:989-1006.

10. Gan SI, Rajan E, Adler DG, et al; ASGE Standards of Practice Committee. Role of EUS. Gastrointest Endosc 2007;66:425-434.

11. Lakhtakia S. Complications of diagnostic and therapeutic endoscopic ultrasound. Best Pract Res Clin Gastroenterol 2016;30:807-823.

12. Eloubeidi MA, Tamhane A, Varadarajulu S, Wilcox CM. Frequency of major complications after EUS-guided FNA of solid pancreatic masses: a prospective evaluation. Gastrointest Endosc 2006;63:622-629.

13. Sato N, Takano S, Yoshitomi H, et al. Needle tract seeding recurrence of pancreatic cancer in the gastric wall with paragastric lymph node metastasis after endoscopic ultrasound-guided fine needle aspiration followed by pancreatectomy: a case report and literature review. BMC Gastroenterol 2020;20:13.

14. Chong A, Venugopal K, Segarajasingam D, Lisewski D. Tumor seeding after EUS-guided FNA of pancreatic tail neoplasia. Gastrointest Endosc 2011;74:933-935.

15. Yane K, Kuwatani M, Yoshida M, et al. Non-negligible rate of needle tract seeding after endoscopic ultrasound-guided fineneedle aspiration for patients undergoing distal pancreatectomy for pancreatic cancer. Dig Endosc 2020;32:801-811.

16. Yokoyama K, Ushio J, Numao N, et al. Esophageal seeding after endoscopic ultrasound-guided fine-needle aspiration of a mediastinal tumor. Endosc Int Open 2017;5:E913-E917.

17. Yamaguchi H, Morisaka H, Sano K, et al. Seeding of a tumor in the gastric wall after endoscopic ultrasound-guided fine-needle aspiration of solid pseudopapillary neoplasm of the pancreas. Intern Med 2020;59:779-782.

18. Minaga K, Kitano M, Enoki E, Kashida H, Kudo M. Needle-tract seeding on the proximal gastric wall after EUS-guided fine-needle aspiration of a pancreatic mass. Am J Gastroenterol 2016;111:1515.

19. Kawabata H, Miyazawa Y, Sato H, et al. Genetic analysis of postoperative recurrence of pancreatic cancer potentially owing to needle tract seeding during EUS-FNB. Endosc Int Open 2019;7:E1768-E1772.

20. Das A, Sivak MV Jr, Chak A. Cervical esophageal perforation during EUS: a national survey. Gastrointest Endosc 2001;53:599-602.

21. Eloubeidi MA, Tamhane A, Lopes TL, Morgan DE, Cerfolio RJ. Cervical esophageal perforations at the time of endoscopic ultrasound: a prospective evaluation of frequency, outcomes, and patient management. Am J Gastroenterol 2009;104:53-56.

22. Grande G, Manno M, Alberghina N, et al. Quick, safe and effective repair of EUS-related duodenal perforation using over-the-scope clip system (with video). Dig Liver Dis 2016;48:1099-1100.

23. Wang KX, Ben QW, Jin ZD, et al. Assessment of morbidity and mortality associated with EUS-guided FNA: a systematic review. Gastrointest Endosc 2011;73:283-290.

24. Facciorusso A, Stasi E, Di Maso M, Serviddio G, Ali Hussein MS, Muscatiello N. Endoscopic ultrasound-guided fine needle aspiration of pancreatic lesions with 22 versus 25 Gauge needles: a meta-analysis. United European Gastroenterol J 2017;5:846-853.
25. Veitch AM, Vanbiervliet G, Gershlick AH, et al. Endoscopy in patients on antiplatelet or anticoagulant therapy, including direct oral anticoagulants: British Society of Gastroenterology (BSG) and European Society of Gastrointestinal Endoscopy (ESGE) guidelines. Endoscopy 2016;48:385-402.

26. Colán-Hernández J, Sendino O, Loras $C$, et al. Antibiotic prophylaxis is not required for endoscopic ultrasonographyguided fine-needle aspiration of pancreatic cystic lesions, based on a randomized trial. Gastroenterology 2020;158:1642-1649.

27. Khashab MA, Chithadi KV, Acosta RD, et al; ASGE Standards of Practice Committee. Antibiotic prophylaxis for GI endoscopy. Gastrointest Endosc 2015;81:81-89.

28. Facciorusso A, Buccino VR, Turco A, Antonino M, Muscatiello N. Antibiotics do not decrease the rate of infection after endoscopic ultrasound fine-needle aspiration of pancreatic cysts. Dig Dis Sci 2019;64:2308-2315.

29. Guarner-Argente C, Shah P, Buchner A, Ahmad NA, Kochman ML, Ginsberg GG. Use of antimicrobials for EUS-guided FNA of pancreatic cysts: a retrospective, comparative analysis. Gastrointest Endosc 2011;74:81-86.

30. Lee LS, Saltzman JR, Bounds BC, Poneros JM, Brugge WR, Thompson CC. EUS-guided fine needle aspiration of pancreatic cysts: a retrospective analysis of complications and their predictors. Clin Gastroenterol Hepatol 2005;3:231-236.

31. Badrinath M, Kanagalingam G, Iorio N, Sapkota B. Are antibiotics necessary for EUS-FNA of pancreatic cysts: a retrospective study: 113. Am J Gastroenterol 2019;114:S69.

32. Papanikolaou IS, Karatzas PS, Triantafyllou K, Adler A. Role of pancreatic endoscopic ultrasonography in 2010. World J Gastrointest Endosc 2010;2:335-343.

33. Savides TJ, Donohue M, Hunt G, et al. EUS-guided FNA diagnostic yield of malignancy in solid pancreatic masses: a benchmark for quality performance measurement. Gastrointest Endosc 2007; 66:277-282.

34. Hewitt MJ, McPhail MJ, Possamai L, Dhar A, Vlavianos P, Monahan KJ. EUS-guided FNA for diagnosis of solid pancreatic neoplasms: a meta-analysis. Gastrointest Endosc 2012;75:319-331.

35. Vazquez-Sequeiros E, Norton ID, Clain JE, et al. Impact of EUSguided fine-needle aspiration on lymph node staging in patients with esophageal carcinoma. Gastrointest Endosc 2001;53:751-757.

36. Parmar KS, Zwischenberger JB, Reeves AL, Waxman I. Clinical impact of endoscopic ultrasound-guided fine needle aspiration of celiac axis lymph nodes (M1a disease) in esophageal cancer. Ann Thorac Surg 2002;73:916-920.

37. Eloubeidi MA, Wallace MB, Reed CE, et al. The utility of EUS and EUS-guided fine needle aspiration in detecting celiac lymph node metastasis in patients with esophageal cancer: a single-center experience. Gastrointest Endosc 2001;54:714-719.

38. Dimitriadis S, Tadic M, Turcic P, et al. EUS-guided fine-needle aspiration (EUS-FNA) in solid pancreatic lesions with 2 passes is equal to 3 passes in collecting adequate sample for diagnosis. Endoscopy 2020;52(S 01):S317-S318.

39. Facciorusso A, Wani S, Triantafyllou K, et al. Comparative accuracy of needle sizes and designs for EUS tissue sampling of solid pancreatic masses: a network meta-analysis. Gastrointest Endosc 2019;90:893-903.

40. Facciorusso A, Sunny SP, Del Prete V, Antonino M, Muscatiello N. Comparison between fine-needle biopsy and fine-needle aspiration for EUS-guided sampling of subepithelial lesions: a meta-analysis. Gastrointest Endosc 2020;91:14-22.

41. Facciorusso A, Del Prete V, Buccino VR, Purohit P, Setia P, Muscatiello N. Diagnostic yield of Franseen and Fork-Tip biopsy needles for endoscopic ultrasound-guided tissue acquisition: a meta-analysis. Endosc Int Open 2019;7:E1221-E1230.

42. Crino SF, Le Grazie M, Manfrin E, et al. Randomized trial 
comparing the fork-tip and the side-fenestrated needles for EUSguided fine-needle biopsy of solid pancreatic lesions. Gastrointest Endosc 2020;92:648-658.e2.

43. Gonçalves B, Soares JB, Bastos P. Endoscopic ultrasound in the diagnosis and staging of pancreatic cancer. GE Port J Gastroenterol 2015;22:161-171.

44. Sharma M, Pathak A, Shoukat A, et al. EUS of the neck: A comprehensive anatomical reference for the staging of head and neck cancer (with videos). Endosc Ultrasound 2019;8:227-234.

45. Uberoi AS, Bhutani MS. Has the role of EUS in rectal cancer staging changed in the last decade? Endosc Ultrasound 2018;7:366-370.

46. Adler DG, Jacobson BC, Davila RE, et al; ASGE. ASGE guideline: complications of EUS. Gastrointest Endosc 2005;61:8-12.

47. Disibeyaz S, Öztaş E, Kuzu UB, Özdemir M. Closure of echoendoscope-related duodenal free wall perforation by placement of a covered metallic stent. Endosc Ultrasound 2016;5:399-400.

48. Liu Y, Wang D, Li Z. Endoscopic closure for EUS and ERCP related duodenal perforation by endoclips. Gastroenterol Res Pract 2016;2016:1051597.

49. Siddiqui UD, Rossi F, Padda MS, Rosenthal LS, Aslanian HR. Patient preferences after endoscopic ultrasound with fine needle aspiration (EUS-FNA) diagnosis of pancreas cancer: rapid communication valued over long-term relationships. Pancreas 2011;40:680-681.

50. Jiang TA, Xie LT. Algorithm for the multidisciplinary management of hemorrhage in EUS-guided drainage for pancreatic fluid collections. World J Clin Cases 2018;6:308-321.

51. Kawakubo K, Yane K, Eto K, et al. A prospective multicenter study evaluating bleeding risk after endoscopic ultrasound-guided fine needle aspiration in patients prescribed antithrombotic agents. Gut Liver 2018;12:353-359.

52. Grassia R, Imperatore N, Capone P, et al. EUS-guided tissue acquisition in chronic pancreatitis: differential diagnosis between pancreatic cancer and pseudotumoral masses using EUS-FNA or core biopsy. Endosc Ultrasound 2020;9:122-129.

53. Burtea D, Dimitriu A, Maloș A, Cherciu I, Săftoiu A. Assessment of the quality of outpatient endoscopic procedures by using a patient satisfaction questionnaire. Curr Health Sci J 2019;45:52-58.

54. Mortensen MB, Fristrup C, Holm FS, et al. Prospective evaluation of patient tolerability, satisfaction with patient information, and complications in endoscopic ultrasonography. Endoscopy 2005;37:146-153. 